\title{
Meta
}

Journal des traducteurs

Translators' Journal

\section{LAFARGA, Francisco, MÉNDEZ, Pedro S. et SAURA, Alfonso, dir. (2007) : Literatura de viajes y traducción. Granada : Editorial Comares, 432 pages}

\section{Montserrat Planelles Iváñez}

Volume 54, numéro 4, décembre 2009

URI : https://id.erudit.org/iderudit/038913ar

DOI : https://doi.org/10.7202/038913ar

Aller au sommaire du numéro

Éditeur(s)

Les Presses de l'Université de Montréal

ISSN

0026-0452 (imprimé)

1492-1421 (numérique)

Découvrir la revue

Citer ce compte rendu

Planelles Iváñez, M. (2009). Compte rendu de [LAFARGA, Francisco, MÉNDEZ, Pedro S. et SAURA, Alfonso, dir. (2007) : Literatura de viajes y traducción.

Granada : Editorial Comares, 432 pages]. Meta, 54(4), 890-890.

https://doi.org/10.7202/038913ar d'utilisation que vous pouvez consulter en ligne.

https://apropos.erudit.org/fr/usagers/politique-dutilisation/ 
decades to consider numerous modes of audiovisual translation from a variety of approaches, opening many avenues for further research.

Christine York

University of Ottawa, Ottawa, Canada

\section{REFERENCE}

Nornes, Abé Mark (1999): For an Abusive Subtitling. Film Quarterly. 52(2):17-34.

Lafarga, Francisco, MÉndez, Pedro S. et Saura, Alfonso, dir. (2007): Literatura de viajes y traducción. Granada: Editorial Comares, 432 pages.

Literatura de viajes y traducción traite de l'intérêt croissant pour la littérature de voyages et des problèmes de traduction qu'elle suscite. Le livre est divisé en 33 chapitres présentés par ordre alphabétique d'auteurs. Toutefois, comme l'indique Francisco Lafarga dans sa présentation, on peut les regrouper par blocs thématiques selon les approches ou les méthodologies utilisées pour le traitement du sujet de la littérature de voyages et de la traduction de celle-ci.

Les auteurs s'entendent pour signaler que les problèmes de traduction les plus fréquents dans ce type de littérature découlent de l'interprétation donnée par le traducteur. En effet, la littérature de voyages présente des problèmes de traduction très précis qui tiennent fondamentalement au traitement des éléments culturels et linguistiques de la part du traducteur, ce dernier se débattant entre la fidélité à la lettre et l'adaptation au contexte d'arrivée.

La présence du traducteur se manifeste de façons diverses: par sa formation, par sa culture, par sa maîtrise de la langue d'origine, par son idéologie ou par ses intentions. Cette approche des problèmes de la traduction de la littérature de voyages centrée sur le traducteur est celle de la majorité des chapitres de ce volume.

La préparation culturelle et historique du traducteur s'avère indispensable pour réussir une traduction fidèle. Dans tous les chapitres concernés, les auteurs s'entendent pour signaler que l'érudition fait partie des compétences du traducteur; les textes analysés sont en effet de précieuses sources de données et de documentation, qui aident le lecteur à mieux comprendre le texte et fournissent au traducteur des connaissances historiques nouvelles. Sarmiento et Batista, par exemple, défendent la traduction philologique au sens strict et soutiennent que, sans le savoir historique et la recherche de sources, il aurait été impossible de traduire les auteurs proposés. Saura montre que le manque d'information et la hâte des éditeurs sont la cause de mauvaises traductions, comme c'est le cas de celle de L'âpre et splendide Espagne de C. Mauclair.

Aguilá et García Garrosa signalent la littéralité et la méconnaissance de la grammaire et de la langue françaises, entre d'autres, comme causes des erreurs et des modifications dans la disposition textuelle - notes et signes de ponctuation -, et Picó y ajoute les difficultés de lecture et de compréhension suscitées par les lettres gothiques, les signes de ponctuation et les faux amis. Solé, pour sa part, attribue les erreurs, les omissions et les modifications de la traduction par rapport au texte de départ à l'âge avancé du traducteur, à son manque de motivation ainsi qu'à son éventuelle incompréhension du texte.

Finalement, les licences du traducteur font l'objet d'étude de plusieurs chapitres. Méndez Robles s'interroge sur les limites de l'intervention du traducteur dans le texte. L'analyse de la traduction de La Havane de la comtesse de Merlin par José Luis Arráez se révèle spécialement intéressante dans la mesure où elle montre les défauts et les omissions d'une traduction contaminée par les intérêts politiques et idéologiques. Iñarrea, pour sa part, expose l'importance des composantes paratextuelles, qui s'avèrent être des sources de connaissance de cultures, d'histoires et de géographies, pour la compréhension d'un texte et pour sa traduction.

Le principal atout de cet ouvrage est de rassembler des études sur la traduction d'un type de littérature trop longtemps ignoré. Aujourd'hui, cependant, la littérature de voyages connaît un regain de popularité spectaculaire et les recherches présentes dans ce volume en sont la preuve palpable.

Nous ignorons les raisons qui ont poussé les éditeurs à présenter les chapitres par ordre alphabétique d'auteurs. Une organisation thématique suivant, par exemple, la classification faite dans la présentation, aurait été préférable pour le confort $\mathrm{du}$ lecteur. On regrettera aussi de ne pas trouver à la fin du volume une bibliographie générale permettant d'accéder à toutes les références utilisées par les auteurs.

Par la richesse et la rigueur des approches des problèmes de traduction présentées, cet ouvrage constitue une source précieuse de réflexions et de références pour tous ceux qui s'intéressent aux problèmes de la traduction de la littérature de voyages.

Montserrat Planelles Iváñez Université d'Alicante, Alicante, Espagne 